Deflagration of HMX-Based Explosives at High Temperatures and Pressures

J. L. Maienschein, J. F. Wardell, M. R. DeHaven, C. K. Black

May 14, 2004

Propellants, Explosives and Pyrotechnics 
This document was prepared as an account of work sponsored by an agency of the United States Government. Neither the United States Government nor the University of California nor any of their employees, makes any warranty, express or implied, or assumes any legal liability or responsibility for the accuracy, completeness, or usefulness of any information, apparatus, product, or process disclosed, or represents that its use would not infringe privately owned rights. Reference herein to any specific commercial product, process, or service by trade name, trademark, manufacturer, or otherwise, does not necessarily constitute or imply its endorsement, recommendation, or favoring by the United States Government or the University of California. The views and opinions of authors expressed herein do not necessarily state or reflect those of the United States Government or the University of California, and shall not be used for advertising or product endorsement purposes. 


\title{
DEFLAGRATION OF HMX-BASED EXPLOSIVES \\ AT HIGH TEMPERATURES AND PRESSURES
}

\author{
J.L. Maienschein, J.F. Wardell, M.R. DeHaven, C.K. Black \\ Lawrence Livermore National Laboratory
}

P.O. Box 808 , L-282

Livermore, CA 94550

\begin{abstract}
We measure the deflagration behavior of energetic materials at extreme conditions (up to $520 \mathrm{~K}$ and $1 \mathrm{GPa}$ ) in the LLNL High Pressure Strand Burner, thereby obtaining reaction rate data for prediction of violence of thermal explosions. The apparatus provides both temporal pressure history and flame time-of-arrival information during deflagration, allowing direct calculation of deflagration rate as a function of pressure. Samples may be heated before testing. Here we report the deflagration behavior of several HMX-based explosives at pressures of 10-600 MPa and temperatures of 300-460 K. We find that formulation details are very important to overall deflagration behavior. Formulations with high binder content $(\geq 15 \mathrm{wt} \%)$ deflagrate smoothly over the entire pressure range regardless of particle size, with a larger particle size distribution leading to a slower reaction. The deflagration follows a power law function with the pressure exponent being unity. Formulations with lower binder content $(\leq 10 \%$ or less by weight) show physical deconsolidation at pressures over 100-200 MPA, with transition to a rapid erratic deflagration 10-100 times faster. High temperatures have a relatively minor effect on the deflagration rate until the HMX $\beta \rightarrow \delta$ phase transition occurs, after which the deflagration rate increases by more than a factor of 10 .
\end{abstract}

Keywords: HMX, deflagration, burn rate, high pressure, thermal damage 


\section{INTRODUCTION}

In considering hazards from energetic materials such as explosives and propellants, thermal response of these materials exposed to high temperatures, as in a fire, is of general interest. When heated to a sufficiently high temperature, energetic materials will produce a thermal explosion. Identifying and understanding the factors determining the violence of the thermal explosion and predicting the violence of thermal explosions remains an challenging goal.

A thermal explosion is a violent event that is initiated by thermal ignition of the energetic charge and subsequently driven by the sub-sonic propagation of a deflagration reaction through the material. (In some very unusual circumstances, a thermally-induced reaction may lead to a detonation, characterized by supersonic propagation of the reaction.) We use the term "deflagration" to indicate the rapid oxidation of the energetic material using oxygen that is part of the molecular structure of the energetic material, as opposed to combustion with an external source of oxygen. During a thermal explosion, the deflagration propagates at high pressures (up to $1 \mathrm{GPa}$ ) and temperatures ( $1000 \mathrm{~K}$ or more). In situations where the heating rate is slow and the energetic material is heated for a prolonged period, thermal degradation prior to the explosion results in the deflagration propagating through thermally-damaged energetic material. The nature of the deflagration process strongly influences the violence of the thermal explosion; materials that exhibit relatively rapid deflagration (particularly in a damaged state) tend to give more violent thermal explosions than those that show relatively slow deflagration behavior.

Experimental characterization of deflagration behavior at high pressure and temperature, for pristine and degraded energetic materials, provides important information on the propagation of the deflagration and the resulting violence of thermal explosions. Here we report experimental characterization of the deflagration behavior of several HMXbased explosives at conditions approaching those present during a thermal explosion, with pressures of 10-600 MPa and temperatures of 300-460 K. We tested both pristine and thermally-damaged materials, the latter involving samples that were held at temperatures up to 420 - $450 \mathrm{~K}$ for periods ranging from a few minutes to over 20 hours. We also draw conclusions concerning the effect of several material variables on deflagration behavior, which may allow extrapolation of results to families of similar materials.

\section{EXPERIMENTS}

\subsection{LLNL HIGH PRESSURE STRAND BURNER}

The LLNL high pressure strand burner, shown schematically in Figure 1, combines the features of a traditional closed-bomb burner with those of a traditional strand burner. The LLNL high-pressure strand burner contains a deflagrating sample in a small volume, high-pressure chamber. We measure temporal pressure data and deflagration front time-of-arrival data to get the laminar deflagration rate for a range of pressures in one experiment. We use a pressure transducer and a load cell to measure the temporal pressure in the bomb, and detect the arrival of the flame front by the burning-through of thin wires embedded in the sample. High speed digital scopes capture the data for subsequent analysis. The combination of pressure and flame front location data allows us to determine if a sample deflagrates in a uniform laminar fashion or if the flame front propagates through in a more erratic nature, for example caused by physical disruption of the sample. This provides a complete picture of the deflagration process, which is otherwise difficult to obtain at these high pressures. In contrast, with a standard closed-bomb burner pressure in the combustion chamber is the only measurement, and calculation of the deflagration rate requires accurate knowledge of the equation of state of the product gases and accurate treatment of heat losses. There is no measure of the surface regression rate to check combustion uniformity, so data from samples that burn erratically are particularly hard to interpret. The standard strand burner provides direct measurement of the surface regression rate in a large volume at constant pressure, giving only one pressure/rate data point in each experiment; furthermore, the large volume required for isobaric operation means that operation at high pressures is generally not practical.

The LLNL high pressure strand burner has a volume of about $75 \mathrm{~cm}^{3}$, and is designed to reach pressures of $1 \mathrm{GPa}$. The pressure vessel body is built from two concentric shells with interference between them to put the inner shell in compression. The shells and the top and bottom end plugs are fabricated from hardened S-5 tool steel, which is suitable for HMX-based explosives which generate little or no corrosive gases. The top end plug is equipped with gas inlet and outlet ports and a pressure transducer, while the bottom end plug holds a pre-wired base and highpressure feed-throughs for the burn wires, ignitor wires, and thermocouples. The pressure transducer is a Kistler model 6213B. For early runs, the load cell was a home-made unit consisting of a $64 \mathrm{~mm}$ long, $16 \mathrm{~mm}$ diameter 
cylinder of 6061-T6 aluminum fitted with a strain gauge and calibrated against the Kistler transducer or another inhouse standard. More recently, the load cell is Omega model LCTB-150K. Both commercial sensors were calibrated to NIST standards by the manufacturer, and in both cases the load cell was calibrated against the Kistler transducer.

The burn sample, shown in Figure 2, is a cylinder $60 \mathrm{~mm}$ long and $6.4 \mathrm{~mm}$ in diameter, made of nine cylindrical pellets stacked on end. Silver burn wires ( $75 \mu \mathrm{m}$ diameter) are inserted between each pair of pellets, in a groove in each pellet. After assembly, the cylindrical surface of the sample is coated with epoxy (Epon 828 with Versamid 140 catalyst) to prevent burning of this surface; this limits the flame front to the end of the cylinder, resulting in a laminar burn. On the upper end of the sample rests the ignitor train: a thin HNS pressed pellet (6.4 mm diameter, 30 $\mathrm{mg}$ ), which is in turn ignited by a hot wire and $130 \mathrm{mg}$ of boron-potassium nitrate. The sample mounts into the prewired base in the bottom end plug. The system is pressurized to the desired starting pressure (5-400 MPa) with argon, and then ignited while recording data. One noteworthy feature is the use of solid argon plugs as the remotelyactuated pressure isolation valves. Once the system is pressurized (using manual valves), it is isolated with solid argon plugs by immersing U-shaped sections of the inlet and outlet tubing in liquid nitrogen. The manual valves are then opened prior to going to remote operation for the ignition and deflagration. After the deflagration is complete, the pressure is released by remotely removing the liquid nitrogen from the outlet tubing. The solid argon plugs provide a reliable remotely-actuated valve for high-pressure gas with no moving parts and no seals to maintain. Additional details on the apparatus have been previously published. [1-3]

Typical pressure and flame front time-of-arrival data are shown in Figure 3. The burn wire electronics provide a well-defined signal, with measured rise times less than 40 microseconds. The wires burn through reproducibly, with wires mounted at the same location in the sample showing a standard deviation of 1-2 milliseconds. The wires do take several milliseconds to burn through; however, this time is essentially independent of initial pressure and temperature conditions and therefore does not affect the deflagration rate calculation made by differences in timesof-arrival. The burn wire at the bottom of the stack does not burn through unless enough energetic material is placed below it to provide several milliseconds of burning once the flame front has passed. Burn wires are recorded in a way that we can unambiguously assign each signal to a particular wire; occasionally a wire will report out of sequence if it is broken by debris in the bomb chamber. The burn wire data should cover the time span of increasing pressure signal - any significant deviation from this indicates anomalous behavior. For example, sometimes the burn wires all report well before the pressure signal reaches its maximum. This indicates that the deflagration front has passed rapidly through the entire sample leaving unreacted material that continues to burn, and is usually interpreted to be the result of physical deconsolidation, i.e. failure of the physical integrity of the sample under pressure. Alternatively, this could represent flame propagation down the side of the sample, although the presence of the epoxy inhibitor should prevent this.

The data in Figure 3 offer a consistency check of the pressure and burn-wire signals. In the experiment shown in Figure 3, the pressure signal increases smoothly and the burn wires report at regularly-spaced intervals, indicating a laminar deflagration. In other runs, we have seen rapid increases in pressure accompanied by burn wires reporting close together followed by more slowly-increasing pressure and further-spaced burn wires. This indicates that one portion of the sample deflagrated faster than the surrounding regions (the reasons for this behavior are unknown). As discussed above, the onset of deconsolidation (physical failure) of a sample is readily apparent in a rapidly-rising pressure and close-reporting burn wires. To calculate deflagration rate as a function of pressure, we use the length and time-of-arrival for each pair of pellets (to smooth the results) and calculate the average pressure for this segment of the sample. The temporal pressure data can be used to calculate the vivacity, $[4,5](1 / P d P / d t)$ although that information is not included here.

For runs with heated samples, the pressure vessel is heated using band heaters to $\sim 400 \mathrm{~K}$. A resistance heater wound into a coil is mounted inside the pressure vessel, surrounding the sample, and is used to heat the sample to the final desired temperature. An internal thermocouple between the sample and the coil heater is used to control this heater. Thermal mapping with an instrumented sample showed that the temperature variation in the top $2 / 3^{\text {rd }}$ of the sample was $\pm 2 \mathrm{~K}$, while the bottom of the sample was $10-15 \mathrm{~K}$ cooler. The top $2 / 3^{\text {rd }}$ of the sample was $5-10 \mathrm{~K}$ hotter than the control thermocouple. In the following discussions of data with heated runs, the specified temperature refers to the control thermocouple reading, since that is all that is available for actual deflagration runs. In runs with no thermal soak, the sample was heated to the final temperature and held for about 10 minutes before ignition. For runs with thermal soak the sample was held at high temperature for 18-22 hours. 


\subsection{MATERIALS}

HMX-based explosives tested in this work are listed in Table 1, along with several formulation details. Representative distributions of particle sizes are shown in Figure 4, and the distribution used in each formulation is shown in Table 1. LX-04 is our baseline explosive, containing $85 \mathrm{wt} \% \mathrm{HMX}$ and $15 \mathrm{wt} \%$ Viton-A, with fine particle "LX-04 grade" HMX (no particles $\geq 300 \mu \mathrm{m}, 90 \% \leq 100 \mu \mathrm{m}$, and 35-50\%<44 $\mu \mathrm{m}$ ). The other formulations represent variations in proportion of HMX, HMX particle size, and in binder composition. Samples were uniaxially pressed in a standard steel die at temperature of $368-378 \mathrm{~K}$ and pressures of $200 \mathrm{MPa}$, and had densities of typically $98 \%$ of the theoretical maximum density for that material.

\section{RESULTS AND DISCUSSION}

\subsection{AMBIENT TEMPERATURE}

Deflagration rate data for LX-04 at $300 \mathrm{~K}$ initial temperature are shown in Figure 5. The combination of fine particle size and high binder content in the baseline LX-04 results in a smoothly-burning laminar deflagration over all pressure conditions at ambient temperature. Two sets of data are included, with pressure measured either by the old load cell or by the pressure transducer. The pressure error bars indicate that the old load cell was less precise than the pressure transducer, so when possible the load cell is not used; however for some runs in the past the pressure transducer was not available. For LX-04, both sets of data are consistent and give a deflagration rate linear in pressure over the pressure range 10-500 MPa. The deflagration rate error bars show that the error is larger at fast rates; there is an inherent noise of $\approx 1-2$ millisecond in the wire time-to-burn, and at the faster rates this becomes a significant contribution to the error. In Figure 5 we show a linear fit of deflagration rate to pressure with the error envelope as dashed lines. If we allowed the pressure exponent to vary, the best value was 0.95 , not significantly different from 1.0, so we chose to use the linear description. The data in Figure 5 are compared with literature data [6-8] for pure HMX (crystals and pressed powder) burn rate in Figure 6. We see that the LX-04 reacts at a somewhat lower rate, as would be expected with its $15 \%$ inert binder. However, the overall agreement demonstrates the validity of this measurement technique.

The deflagration behavior of LX-11 and RX-04-AN are shown in Figure 7. LX-11, which contains more binder ( $20 \%$ vs $15 \%$ ) but is otherwise identical to LX-04, exhibited very similar behavior to LX-04, with perhaps a slightly reduced deflagration rate, although we did not study it over as wide a range of conditions. RX-04-AN has the same composition as LX-04, but with a larger particle size distribution (Table 1); measurements with RX-04-AN were designed to determine if the presence of large particles in the HMX leads to rapid deflagration through physical failure of the samples by crystal fracture. As seen in Figure 7, this was not the case - this material showed uniform and regular deflagration over the entire pressure range, albeit with a slower rate than LX-04. This slower rate is expected, and indicates that the large crystals remain intact during deflagration - larger particles have smaller surface-to-volume ratios, and deflagration occurs at the crystal surface as long as the crystal remains intact.

For formulations with the same ingredients but lower in binder (LX-07, Figure 8 and LX-10, Figure 9), the deflagration is very similar to that of LX-04 at lower pressure. However, at pressures above $\sim 150 \mathrm{MPa}$ the deflagration becomes very rapid and erratic, increasing 10-100-fold in some segments of the sample as measured by burn wire data. In many of these runs the flame front passed through the sample well before the entire sample was consumed. This behavior, called deconsolidative burning, represents the loss of physical integrity of the material at high pressure, with the resultant fracturing, formation of small particles with high surface area, and rapid deflagration through the increased-porosity sample. $[6,9,10]$ Deconsolidative burning is seen at lower pressures with pressed samples of binderless HMX powder; $[6,9]$ it is reasonable to expect it at higher pressures for plasticbonded explosives that are more physically robust. From these data we conclude that a binder content of $>10 \%$ by weight is needed to avoid deconsolidative burning, at least for the Viton A binder used here. We note that the deflagration rate of LX-10 is essentially the same as LX-04 at low pressures, and is significantly higher than that of RX-04-AN, which has the same particle size distribution as LX-10 and the same composition as LX-04. Apparently the slowing of deflagration in LX-10 by the presence of larger particles is coincidentally offset by the effect of the higher HMX content when compared to LX-04. 
PBX-9501, which has a high HMX content (95 weight \%) and a binder (Estane) heavily plasticized with an energetic plasticizer, shows similar behavior to that of LX-07 and LX-10 (Figure 10). Here the binder composition is quite different, but apparently the low binder content dominates the behavior and the onset of physical deconsolidation. The presence of the energetic binder does not significantly increase the deflagration rate over that of LX-10 or LX-07.

\subsection{HIGH TEMPERATURE}

Our measurements with heated samples were made with LX-04, because its uniform behavior at ambient temperature simplifies the identification of temperature effects. The deflagration behavior of heated LX-04 is shown in Figure 11, for samples that were pressurized to the starting pressure while at ambient temperature, then heated to the final temperature and ignited. For initial pressures over $100 \mathrm{MPa}$, the deflagration rates are perhaps slightly higher for the hot samples than for the ambient temperature samples, and the same is true for low initial pressures and a temperature of $423 \mathrm{~K}$. Based on HMX thermal decomposition data,[11-13] we would not expect significant thermal degradation under these conditions, so these results represent the effect of temperature on deflagration.

For LX-04 samples at low initial pressures and a temperature of $440 \mathrm{~K}$, the deflagration behavior is much faster and erratic, indicative of deconsolidation. This is explained by the $\beta \rightarrow \delta$ solid-solid phase transition in HMX. The onset temperature for this phase conversion at ambient pressure is $\sim 433 \mathrm{~K}$, [14-18] but because the density decreases about $8 \%$ with phase conversion,[19] the transition temperature increases as pressure increases.[20, 21] At 100 MPa the phase transition temperature increases to about 480-490K.[20] Therefore, in the experiments in Figure 11 which were pressurized to $>100 \mathrm{MPa}$ before heating, the phase conversion was prevented during the subsequent heating to $440 \mathrm{~K}^{\circ} \mathrm{C}$; the data represent the deflagration of LX-04 with beta-phase HMX at the reported temperatures. Similarly, for the experiments in Figure 11 with low initial pressures and initial temperature of $423 \mathrm{~K}$, the HMX remained in beta phase. However, for the experiments with low initial pressure and initial temperature of $440 \mathrm{~K}$, the HMX did apparently convert to delta phase with the corresponding volumetric increase. The volume expansion of the randomly-oriented HMX crystals leads to irregular growth and physical disruption of the sample, with loss of physical integrity and formation of increased surface area. This, in turn, leads to deconsolidative deflagration, similar to that seen in low-binder formulations at high pressures and ambient temperature. A possible additional factor could be that $\delta$-phase HMX has an inherently-higher deflagration rate; however this is entirely speculative and would require measurements with fully-dense samples of $\delta$-phase HMX.

The effect of thermal damage from prolonged heating of LX-04 is shown in Figure 12. Samples heated for 22 hours at temperature $<433 \mathrm{~K}$ show somewhat increased deflagration rates (about 3-fold faster), which result from thermal degradation since this temperature is too low to drive the phase conversion. A sample heated for 22 hours at $453 \mathrm{~K}$ at $160 \mathrm{MPa}$ showed deflagration behavior similar to that of samples heated at $<433 \mathrm{~K}$, indicating that the effect of thermal damage was only slightly increased by the $20 \mathrm{~K}$ higher temperature. A sample heated for 22 hours at $453 \mathrm{~K}$ at $100 \mathrm{MPa}$ showed rapid deflagration at the beginning of the run and a rapidly decreasing deflagration rate as the reaction proceeded (despite an increase in pressure). This may indicate that the sample was just beginning to phase convert, and that the increase in pressure during deflagration was sufficient to reverse the phase conversion during the experiment. The latter part of that interpretation is speculative, however; another explanation is that the temperature gradient along the sample led to phase conversion at the top where it was ignited, while the cooler lower region did not undergo phase conversion. We do not have sufficient data to distinguish between these explanantions. Finally, many runs were made with samples heated for 18-22 hours at temperature above $433 \mathrm{~K}$ at ambient pressure, These all showed greatly-increased deflagration rates (about 40-fold faster), resulting from the phase transition and loss of physical integrity of the samples.

\section{SUMMARY OF RESULTS}

\subsection{AMBIENT TEMPERATURE RESULTS}

The deflagration rate data reported here, taken with several different HMX formulations over a wide range of pressures and temperatures, allow identification of key factors controlling deflagration behavior. LX-04 provides an excellent baseline behavior - its small particle size distribution and high binder content result in uniform and regular deflagration, with the first-order rate expression 


$$
\text { deflagration rate }(\mathrm{m} / \mathrm{s})=0.97 \times 10^{-3} \mathrm{P}(\mathrm{MPa}) .
$$

Formulation variations from this material provide insight into the importance of different factors. The key formulation variable is the quantity of binder present - formulations with less than $15 \%$ binder by weight exhibit very rapid deconsolidative deflagration at pressures above 100-150 MPa. The HMX particle size and the HMX content also play a role, with larger particles and lower HMX content giving slower deflagrations rates. We could not distinguish a difference between an inert and reactive binder at ambient temperature.

\subsection{ELEVATED TEMPERATURE RESULTS}

Our complete results (including some not in Figure 11 or 12) are summarized in Table 2. The deflagration rate of LX-04 at temperatures up to $453 \mathrm{~K}$ with no prolonged exposure to high temperature and no phase conversion is little-changed from that at ambient temperature; in the runs at $453 \mathrm{~K}$ with no soak there was insufficient time for the phase conversion to take place, so the samples were tested in the initial beta phase. With prolonged heating in the absence of phase conversion, thermal damage leads to increased deflagration rates, about 3 times faster after 22 hours at $423-428 \mathrm{~K}$ and about 10 times faster at $453 \mathrm{~K}$. The $\beta \rightarrow \delta$ phase transition has the biggest effect on deflagration behavior, with an approximately 40-fold increase following phase conversion.

\subsection{EXAMINATION OF TEMPORAL PRESSURE DATA}

The pressure-time data collected for each experiment provides additional information on the deflagration process. For example, erratic or very rapid pressure increases indicate deconsolidative burning. Inasmuch as the increased reaction rate during deconsolidative deflagration represents an increase in burning surface area from physical fracture, comparison of the rates of pressure increase for deconsolidative and uniform deflagration allows a quantification of the increase in surface area resulting from deconsolidation (following the well-known vivacity concept in combustion $[4,5])$. These analyses are underway for the experiments reported here, and will be the subject of a future paper.

\section{CONCLUSIONS}

We have characterized the deflagration behavior of HMX-based explosives by measurement in a high pressure hybrid strand burner. Through measurements on LX-04, a robust HMX-Viton-A formulation, we have established an inherent deflagration rate of HMX formulations. We have also identified variations driven by formulation details, which in some cases lead to erratic and greatly-accelerated deflagration rates. Low binder content results in physical failure of the sample with a concomitant increase in surface area and deflagration rate. Heating $>440 \mathrm{~K}$ at low pressure causes the $\beta \rightarrow \delta$ phase transition, which physically disrupts the sample and again results in a very fast deflagration. Both of these mechanisms are physical in nature, not chemical, but both will certainly play a significant role in the overall violence of a thermal explosion. In contrast, thermal degradation of HMX at temperatures up to $455 \mathrm{~K}$ appears to play a minor role in the deflagration process.

In a thermal explosion the reaction propagates by deflagration at high pressures. In cases with slow heating rates, the deflagration is propagating in heated explosive, while in cases with fast heating rates much of the reaction will propagate in unheated explosive. Therefore, characterization of the high-pressure deflagration behavior of explosives at both ambient and elevated temperatures provides insight into the propagation of thermal explosions in those same materials.

\section{ACKNOWLEDGEMENTS}

We gratefully acknowledge financial support for this work by the LLNL High Explosives Surety Project and the Joint DoD/DOE Munitions Technology Development Program. Key contributors to this experimental effort include Les Calloway, Kou Moa, and Greg Sykora. We also thank Al Nichols, Jack Reaugh, and Matthew McClelland for fruitful discussions of the work as it progressed.

This work was performed under the auspices of the U.S. Department of Energy by the University of California, Lawrence Livermore National Laboratory under contract number W-7405-Eng-48. 


\section{REFERENCES}

1. J.L. Maienschein and J.B. Chandler, High Pressure Laminar Burn Rates of AP/A1/HTPB Propellants, Proceedings of JANNAF 34th Combustion and 16th Propulsion Systems Hazards Subcommittee Meetings, West Palm Beach, FL, CPIA, Publication 657, Vol. II, p. 951997.

2. J.L. Maienschein and J.B. Chandler, Burn Rates of Pristine and Degraded Explosives at Elevated Pressures and Temperatures, Proceedings of 11 th International Detonation Symposium, Snowmass, CO, Office of Naval Research, ONR 33300-5, p. 8721998.

3. J.L. Maienschein and J.F. Wardell, Deflagration Behavior of PBXN-109 and Composition B at High Pressures and Temperatures, Proceedings of JANNAF 38th Combustion and 20th Propulsion Systems Hazards Subcommittee Meetings, Sandestin, FL, CPIA, Publication JSC CD-14 2002.

4. A. Birk, D.E. Kooker and P. Baker, Model of Cavity Combustion Within an Energetic Solid: Application to Composition-B, Proceedings of JANNAF 37th Combustion and 19th Propulsion Systems Hazards Subcommittee Meetings, Monterey, CA, CPIA, Publication 704, Vol. II, p. 952000.

5. R. Lieb and P. Baker, Combustion Morphology of TNT and Composition B, Proceedings of JANNAF 37th Combustion and 19th Propulsion Systems Hazards Subcommittee Meetings, Monterey, Ca, CPIA, Publication 704, Vol. II, p. 812000.

6. T.L. Boggs, Thermal Behavior of Cyclotrimethylenetrinitramine(RDX) and Cyclotetramethylenetetranitramine (HMX), in K.S. Kuo and M. Summerfield (Eds), Fundamentals of Solid-Propellant Combustion, American Institute of Aeronautics and Astronautics, New York 1984.

7. R.L. Derr, T.L. Boggs, D.E. Zurn and E.J. Dibble, The Combustion Characteristics of HMX, Proceedings of 11th JANNAF Combustion Meeting, Pasadena, CA, CPIA, Pub. 261, p. 2311974.

8. M. Herrmann, W. Engel and N. Eisenreich, Thermal Expansion, Transitions, Sensitivities and Burning Rates of HMX, Propellants, Explosives, Pyrotechnics 1992 17, 190.

9. R.A. Fifer and J.E. Cole, Transitions from Laminar Burning for Porous Crystalline Explosives, Proceedings of the Seventh Symposium (International) of Detonation, Annapolis, MD, Office of Naval Research, NSWC MP-82-334, p. 1641981.

10. W.C. Tao, M.S. Costantino and D.L. Ornellas, Burning Rates of Two Cast Nitramine Explosives Using a Hybrid Closed Bomb-Strand Burner, Proceedings of the Ninth Symposium (International) of Detonation, Portland, OR, Office of the Chief of Naval Research, OCNR 113291-7, p. 13101989.

11. R. Behrens, Thermal decomposition of HMX and RDX: decomposition processes and mechanisms based on STMBMS and TOF velocity-spectra measurements, in S.N. Bulusu (Ed), Chemistry and Physics of Energetic Materials, Kluwer Academic Publishers, the Netherlands 1990.

12. R. Behrens, Thermal decomposition of energetic materials: temporal behaviors of the rates of formation of the gaseous products from condensed-phase decomposition of octahydro-1,3,5,7-tetranitro-1,3,5,7-tetrazocine, J. Phys. Chem., 1990 94, 6706.

13. R. Behrens, Identification of octahydro-1,3,5,6-tetranitro-1,3,5,7-tetrazocine (HMX) pyrolysis products by simultaneous thermogravimetric modulated beam mass spectrometry and time-of-flight velocity-spectra measurements, Int. J. Chem. Kin., 1990 22, 135.

14. H.H. Cady, A.C. Larson and D.T. Cromer, The crystal structure of alpha-HMX and a refinement of the structure of beta-HMX, Acta Cryst., 1963 16, 617. 
15. T.B. Brill and R.J. Karpowicz, Solid phase transition kinetics: the role of intermolecular forces in the condensed phase decomposition of octahydro-1,3,5,7-tetranitro-1,3,5,7-tetrazocine, J. Phys. Chem., 1982 86, 4260.

16. T.B. Brill and C.O. Reese, Analysis of intermolecular interactions relating in the thermophysical behavior of alpha, beta, and delta octahydro-1,3,5,7-tetranitro-1,3,5,7-tetrazocine, J. Phys. Chem, 198084.

17. W.C. McCrone, Crystallographic Data: Cyclotetramethylene Tetranitramine (HMX), Analytical Chem., 1950 22, 1225.

18. A.S. Teetsov and W.C. McCrone, The Microscopial Study of Polymorph Stability Diagrams, Microscop. Cryst. Front., 1965 15, 13.

19. H.H. Cady, Studies on the Polymorphs of HMX, Report LAMS-2652, 1961, Los Alamos Scientific Laboratory, Los Alamos, NM, USA.

20. R.J. Karpowicz and T.B. Brill, The $\beta \rightarrow \delta$ Transformation of HMX: Its Thermal Analysis and Relationship to Propellants, AIAA Journal, 1982 20, 1586.

21. A.G. Landers and T.B. Brill, Pressure-Temperature Dependence of the $\beta \rightarrow \delta$ Polymorph Interconversion in Octahydro-1,3,5,7-tetranitro-1,3,5,7-tetrazocine, J. Phys. Chem, 1980 84, 3573. 
Table 1. HMX-based explosives for which deflagration measurements are reported

\begin{tabular}{|l|c|c|c|c|}
\hline Material & $\begin{array}{c}\text { Wt. \% } \\
\text { HMX }\end{array}$ & Binder & $\begin{array}{c}\text { HMX particle } \\
\text { size distribution }\end{array}$ & $\begin{array}{c}\text { Typical density (98\% of theoretical } \\
\text { maximum) }\end{array}$ \\
\hline LX-11 & 80 & Viton A & "LX-04" & 1.85 \\
LX-04 & 85 & Viton A & "LX-04" & 1.85 \\
RX-04-AN & 85 & Viton A & "LX-10" & 1.85 \\
LX-07 & 90 & Viton A & "LX-04" & 1.85 \\
LX-10 & 94.5 & Viton A & "LX-10" & 1.86 \\
PBX-9501 & 95 & $50 \%$ Estane, 50\% & "PBX-9501" & 1.82 \\
& & BDNPA/F & & \\
\hline
\end{tabular}


Table 2. Summary of the effect of heating on deflagration behavior of LX-04. "Normalized rate" is referenced to LX-04 deflagration rate at ambient temperature.

\begin{tabular}{|c|c|c|c|c|c|c|}
\hline $\begin{array}{c}\text { Initial deflagration } \\
\text { pressure, MPa }\end{array}$ & $\begin{array}{c}\text { Temp., } \\
\mathrm{K}\end{array}$ & $\begin{array}{c}\text { Soak time, } \\
\text { hours }\end{array}$ & $\begin{array}{c}\text { Soak P, } \\
\text { MPa }\end{array}$ & $\begin{array}{c}\text { Phase } \\
\text { change? }\end{array}$ & $\begin{array}{c}\text { Replicates } \\
\text { rormalized } \\
\text { rate }\end{array}$ \\
\hline $10-250$ & $423-433$ & 0 & - & No & 14 & 1 \\
$100-250$ & $423-428$ & $18-22$ & $0.1-150$ & No & 7 & 3 \\
$100-265$ & 453 & 0 & - & No & 8 & 1 \\
$130-160$ & 453 & $18-22$ & $130-160$ & No & 2 & 10 \\
130 & 453 & $18-22$ & 0.1 & Yes & 6 & 40 \\
\hline
\end{tabular}




\section{Figure Captions}

Figure 1. Schematic of strand burner. a - nine-segment burn sample, with burn wires between segments ( only two wires shown for clarity) and ignitor on top; $b$ - top plug with inlet and outlet ports and pressure transducer in center; c - load cell; d - pressure vessel; e - bottom plug with wire feed-throughs; $\mathrm{f}$ - signal wires to electronics; $\mathrm{g}$ - load frame (top and bottom)

Figure 2. Sample holder and explosive sample. Sample is between two vertical posts, with burn wires between pellets, and is covered with epoxy. $\mathrm{BKNO}_{3} / \mathrm{HNS}$ ignitor in cardboard tube is on top, with ignition wires leading to it. Thermocouple at right is used to monitor temperature inside pressure vessel.

Figure 3. Typical data from strand burner, showing temporal pressure behavior and flame-front time-of-arrival signals. Each time-of-arrival signal has unique identifier. Wires 1 (at ignitor), 4, 8 - solid line, wires 2, 5, 9 - shortdashed line, wires 3, 7, 10 - long dashed line, each with first wire in set at maximum amplitude, second wire at minimum amplitude, and third wire at intermediate amplitude. Wire 6 - dash-dot line at full amplitude.

Figure 4. Particle size distribution of different HMX formulations

Figure 5. LX-04 deflagration rate data at 300K, with linear fit to data. Dashed lines represent range of fit at $95 \%$ confidence level.

Figure 6. Comparison of LX-04 results with literature HMX data

Figure 7. Deflagration rate data for LX-11, and RX-04-AN, with fit to LX-04 data shown for comparison

Figure 8. Deflagration rate data for LX-07, with fit to LX-04 data shown for comparison

Figure 9. Deflagration rate data for LX-10, with fit to LX-04 data shown for comparison

Figure 10. Deflagration rate data for PBX-9501, with fit to LX-04 data shown for comparison

Figure 11. Deflagration rate data for heated LX-04 with no thermal damage (no soak)

Figure 12. Deflagration rate data for heated and thermally-damaged LX-04 


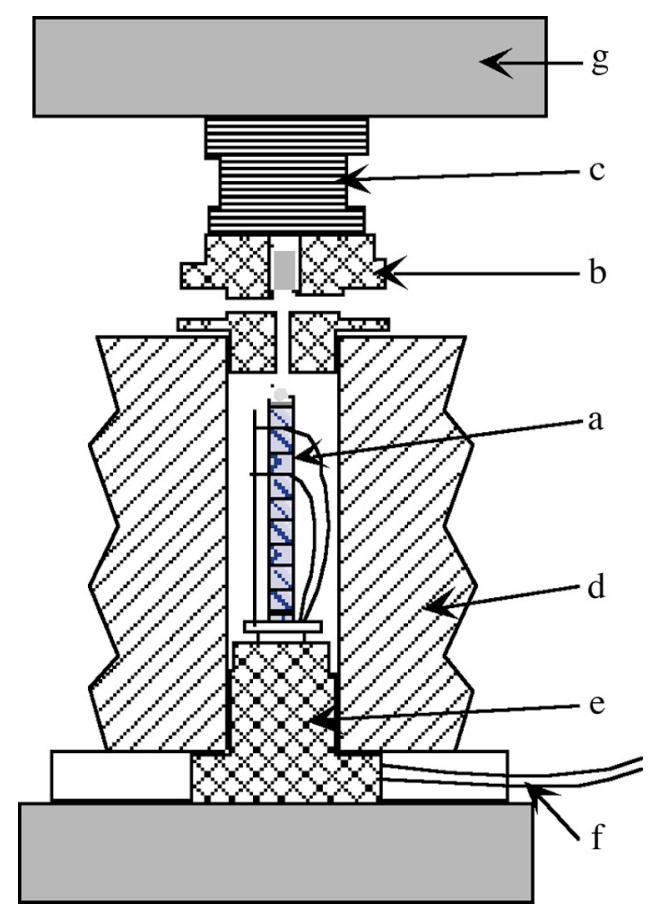

Figure 1 


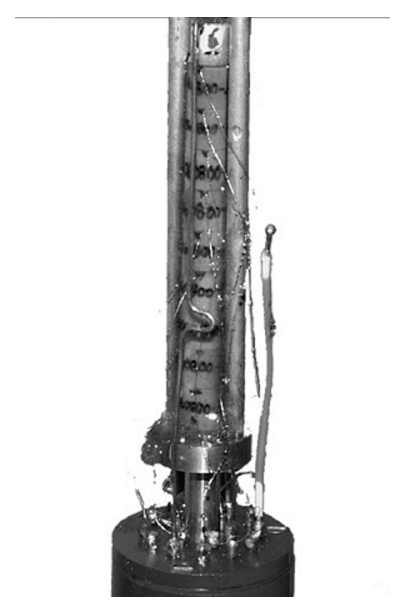

Figure 2 


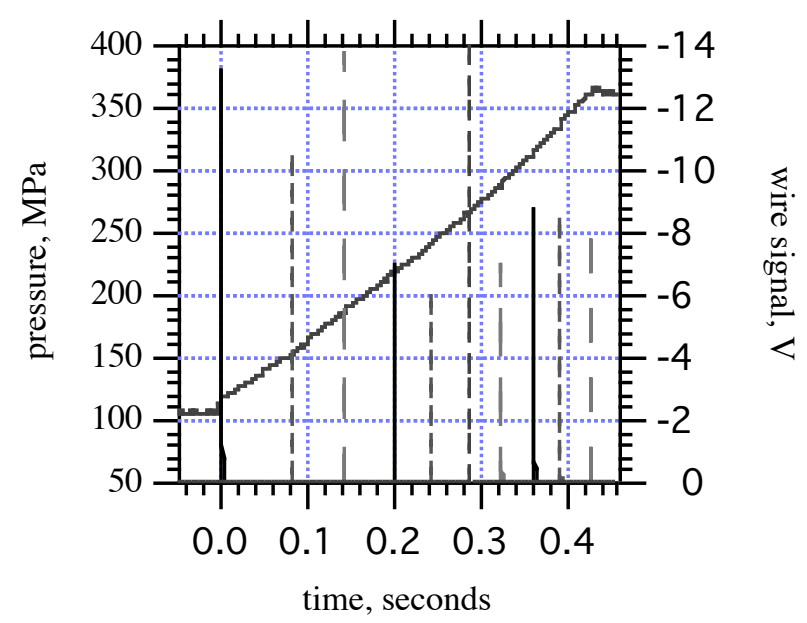

Figure 3 


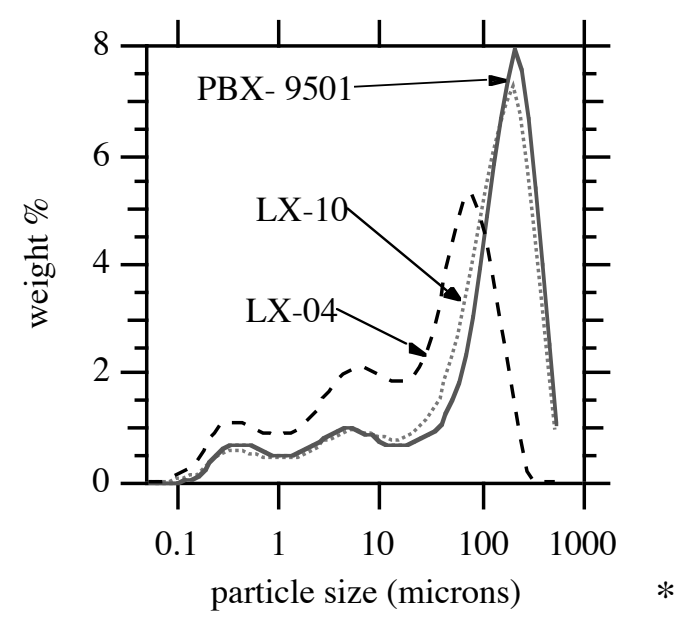

Figure 4 


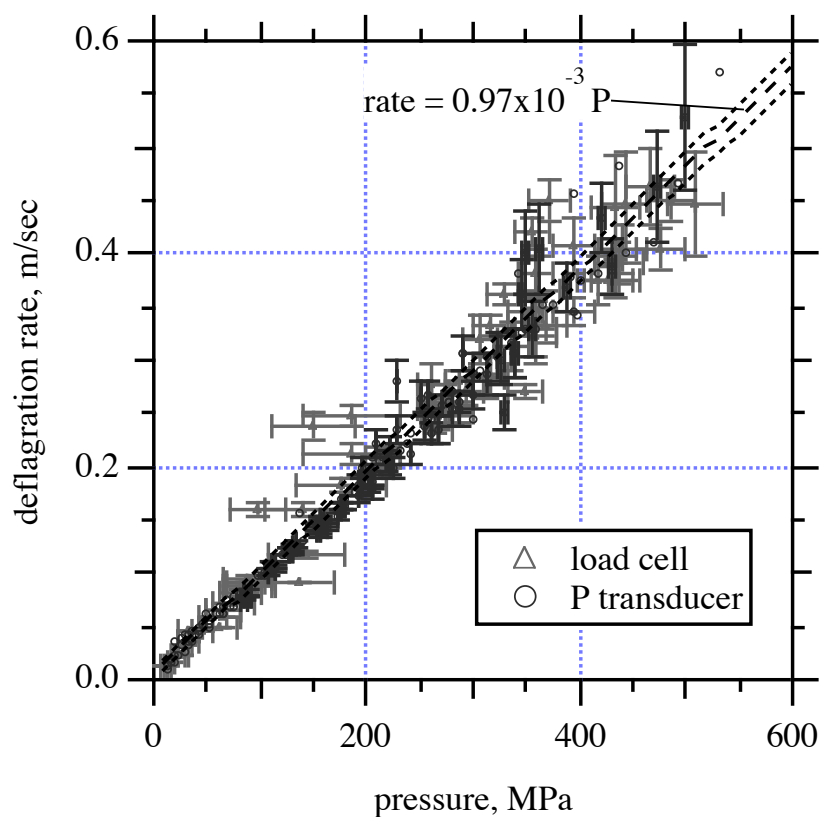

Figure 5 


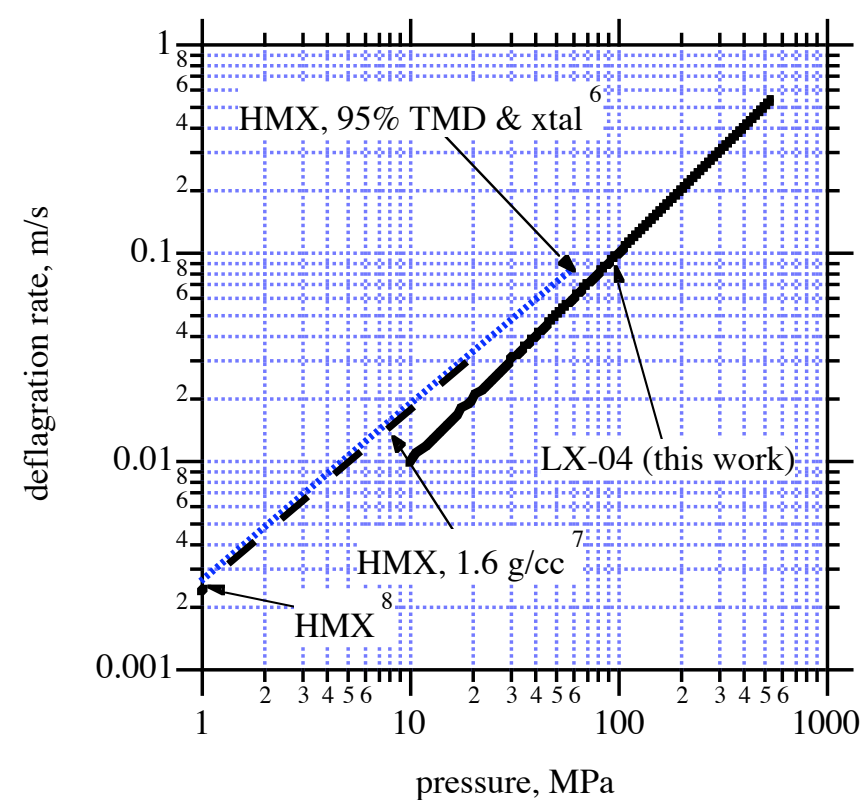

Figure 6 


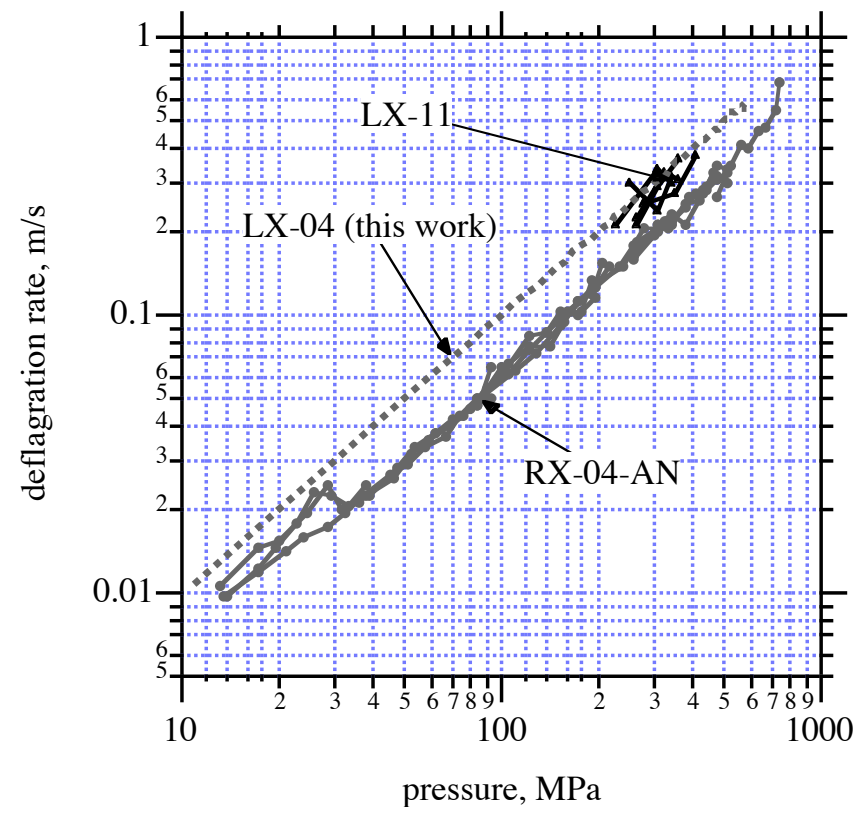

Figure 7 


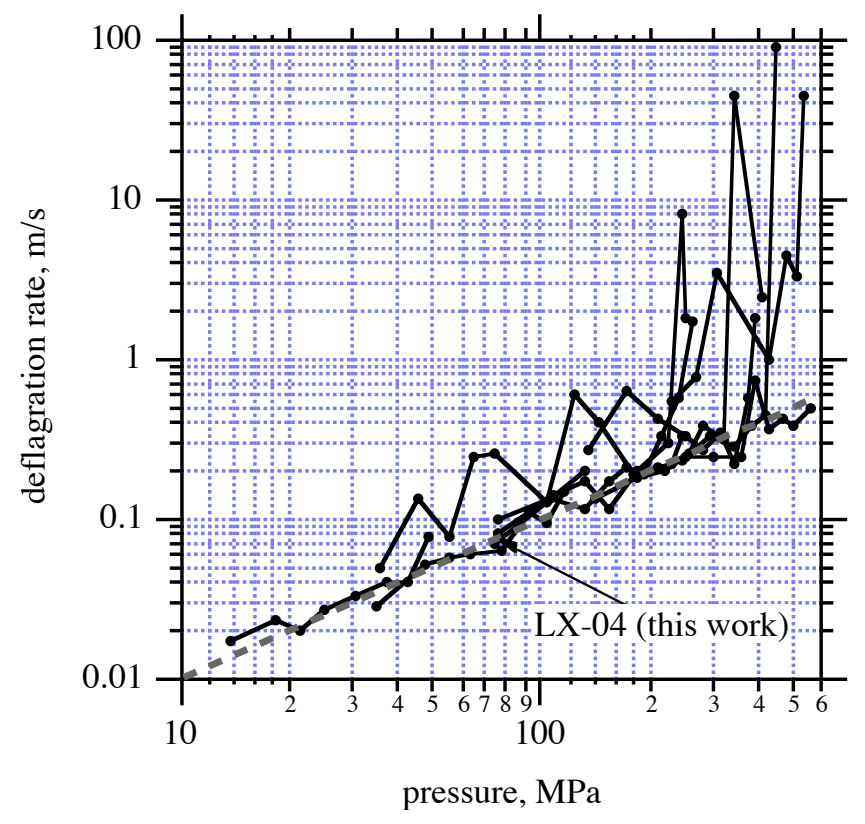

Figure 8 


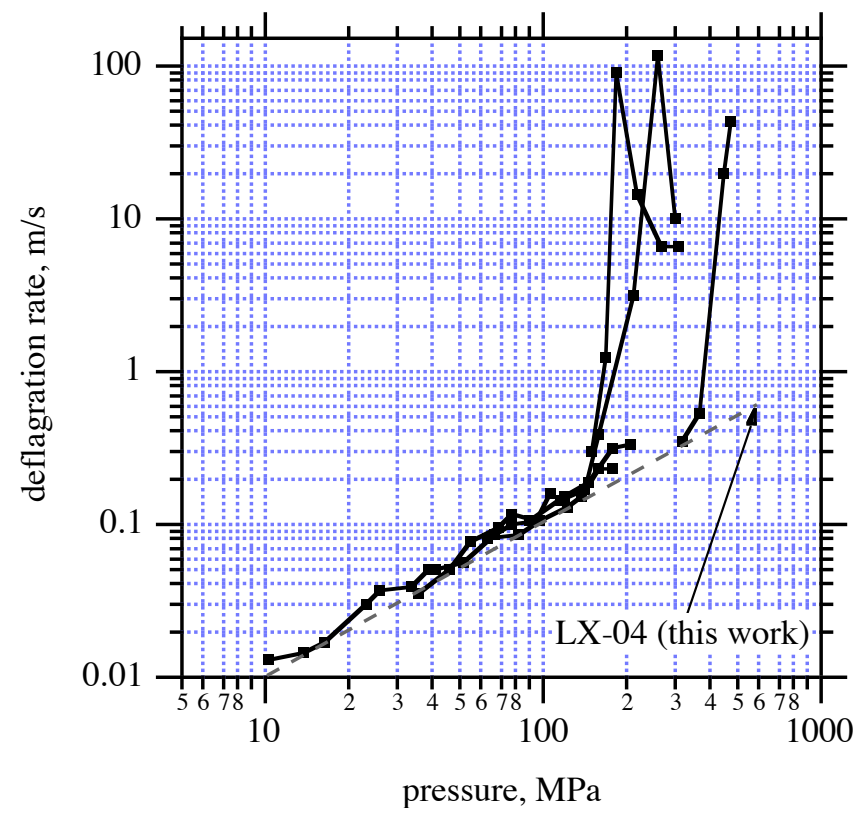

Figure 9 


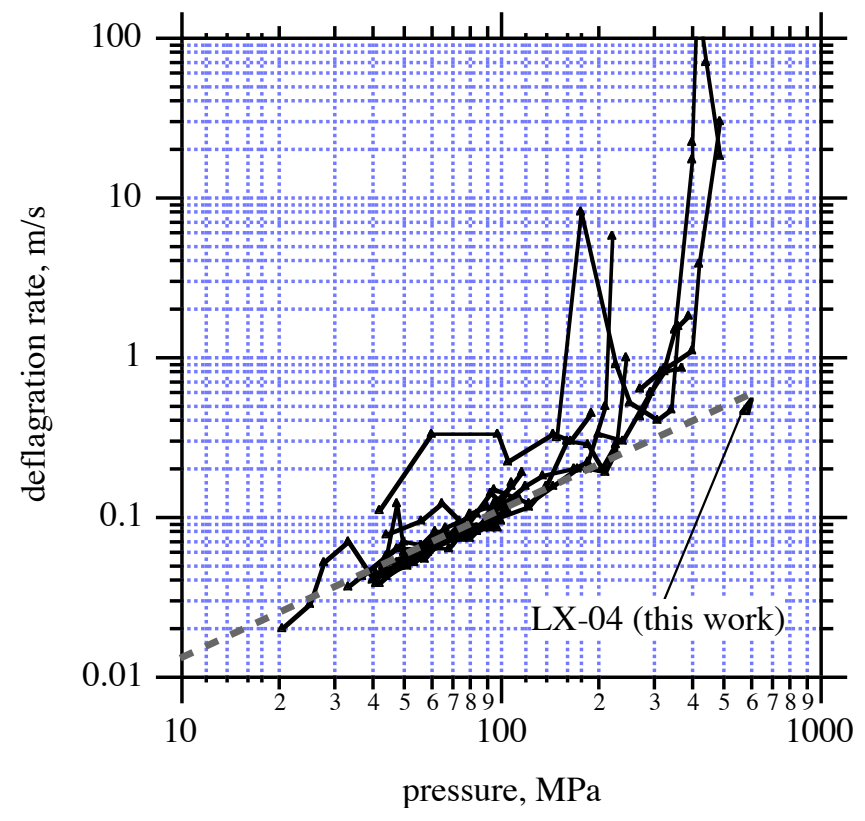

Figure 10 


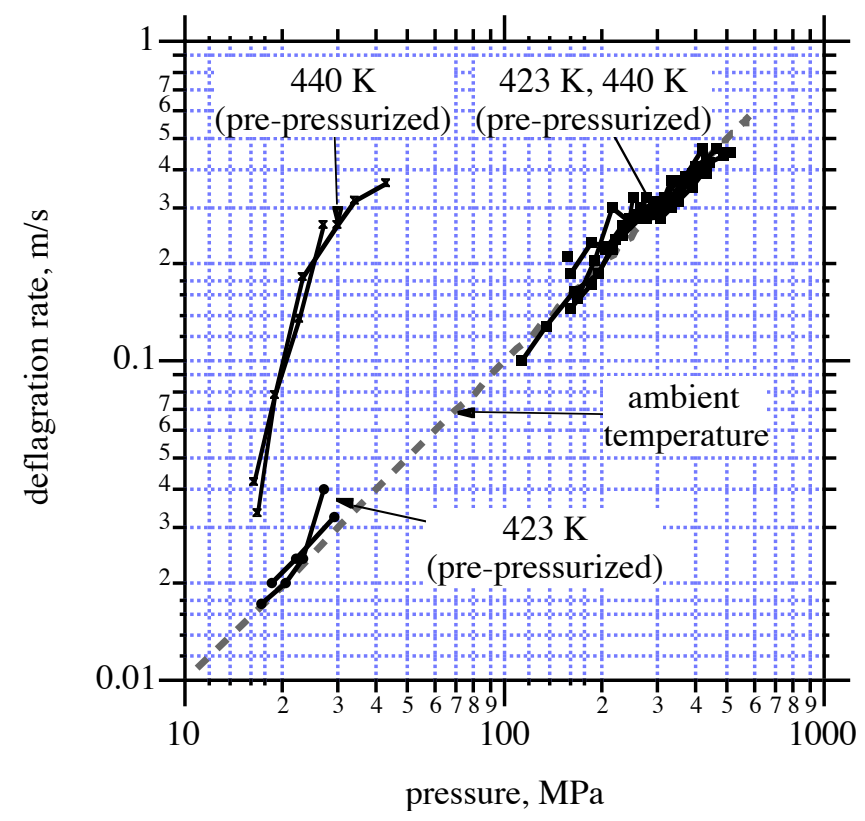

Figure 11 


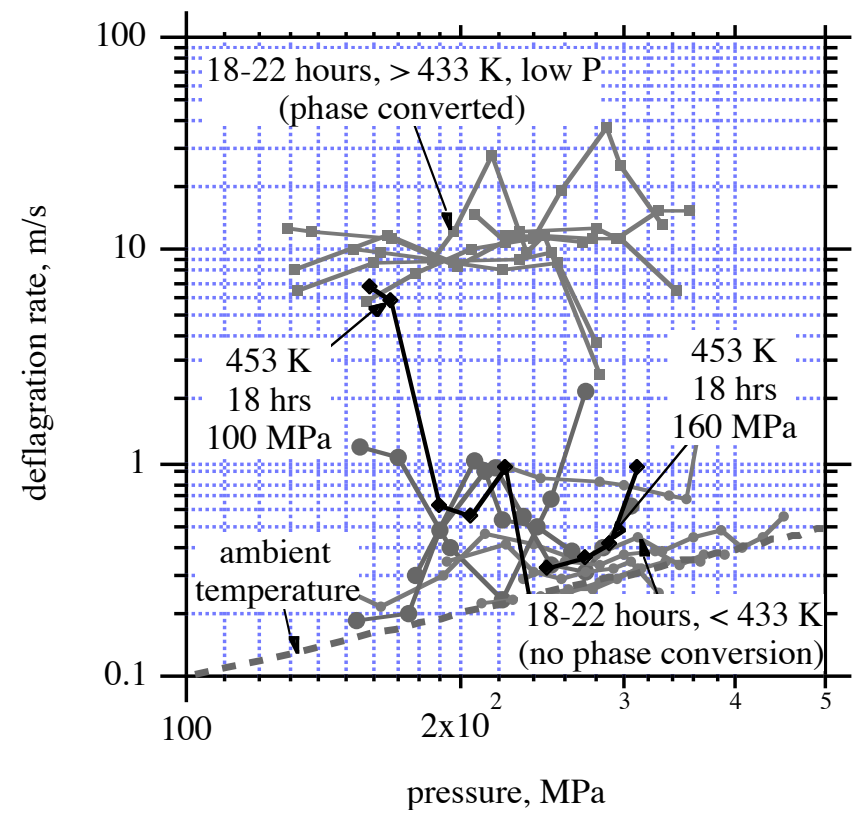

Figure 12 